\title{
Solubilidad del colágeno y textura de la carne bovina cocinada al vacío: efecto del tiempo de maduración y de la temperatura de cocinado
}

\author{
B. Panea* y G. Ripoll
}

Centro de Investigación y Tecnología Agroalimentaria de Aragón-Instituto Agroalimentario de Aragón (CITA-IA2). Avenida de Montañana 930, 50059, Zaragoza, España

\section{Resumen}

La cocina al vacío se está utilizando de manera creciente en la restauración porque presenta varias ventajas sobre el cocinado tradicional pero tanto la presión de vacío como la temperatura de cocinado condicionan la textura de la carne. Este trabajo estudia, en dos razas bovinas de aptitudes diferentes, los cambios que ocurren en la textura de la carne y la solubilidad del colágeno en función de la temperatura de cocinado y el tiempo de maduración. Se utilizaron 13 animales de raza Pirenaica y 12 de raza Frisona. El músculo Longissimus lumborum et thoracis se fileteó siguiendo un diseño factorial con tres tiempos de maduración ( 7,14 y 21 días) y 4 temperaturas de cocinado para la textura (crudo, $55^{\circ} \mathrm{C}, 65^{\circ} \mathrm{C}$ y $70^{\circ} \mathrm{C}$ ), o 3 temperaturas para el colágeno insoluble y las pérdidas por cocinado $\left(55^{\circ} \mathrm{C}, 65^{\circ} \mathrm{C}\right.$ y $\left.70^{\circ} \mathrm{C}\right)$. La maduración tuvo un menor efecto que la temperatura de cocinado sobre la textura de la carne. El porcentaje de pérdidas por cocinado aumentó con la temperatura. La solubilidad del colágeno se vio poco afectada por la temperatura en el rango $50^{\circ} \mathrm{C}-70^{\circ} \mathrm{C}$. En general, la dureza del músculo fue mayor en crudo que cocinada a $55^{\circ} \mathrm{C}$, y se fue incrementando con la temperatura. A la vista de los resultados, no recomendaríamos cocinar la carne por encima de $65^{\circ} \mathrm{C}$, ya que por encima de esta temperatura se ve comprometida la textura.

Palabras clave: Dureza, maduración, solubilidad, temperatura, vacuno.

Collagen solubility and beef meat texture under vacuum-cooking: effect of cooking temperature and ageing time

\begin{abstract}
Sous-vide or under-vacuum cooking is being used increasingly in restoration because it has several advantages, but both vacuum pressure and cooking temperature affect meat texture. This paper investigates, in two cattle breeds of different aptitudes, meat texture and collagen solubility in function of the cooking temperature and ageing time. 13 animals from Pirenaica breed and 12 from Frisona breed were used. Muscle Longissimus thoracis et lumborum was chopped following a design of three ageing times (7, 14 and 21 days) and 3 or 4 cooking temperatures: raw, $55^{\circ} \mathrm{C}, 65^{\circ} \mathrm{C}$ and $70^{\circ} \mathrm{C}$ for texture; $55^{\circ} \mathrm{C}$, $65^{\circ} \mathrm{C}$ and $70^{\circ} \mathrm{C}$ for insoluble collagen and cooking losses. Ageing had less effect than temperature on meat texture. The percentage of cooking losses increased as temperature did. The solubility of collagen was slightly affected by the temperature in the range $50^{\circ} \mathrm{C}-70^{\circ} \mathrm{C}$. In the range $50^{\circ} \mathrm{C}-70^{\circ} \mathrm{C}$ muscles suffered great conformational changes. In general, toughness decreased until to $55^{\circ} \mathrm{C}$ and increased slightly since then. In view of the results, we would not recommend cooking the meat above $65^{\circ} \mathrm{C}$ since above it texture would be adversely affected.
\end{abstract}

Keywords: Toughness, ageing, solubility, temperature, beef.

\footnotetext{
* Autor para correspondencia: bpanea@aragon.es
}

Cita del artículo: Panea B, Ripoll G (2019). Solubilidad del colágeno y textura de la carne bovina cocinada al vacío: efecto del tiempo de maduración y de la temperatura de cocinado. ITEA-Información Técnica Económica Agraria 115(2): 163-174. https://doi.org/10.12706/itea.2018.034 


\section{Introducción}

La cocina al vacío se está utilizando de manera creciente en la restauración porque presenta varias ventajas: facilita la logística de la cocina, mejora las condiciones higiénico-sanitarias al reducir el procesado y manipulación y aporta texturas nuevas, posibilitando la creación de nuevas recetas. La cocina al vacío tiene dos características fundamentales: 1. el alimento está sometido a una presión de vacío y 2. se controla escrupulosamente la temperatura de cocinado. Pero tanto la presión de vacío (Baldwin, 2012) como la temperatura de cocinado condicionan enormemente la textura de la carne (Combes et al., 2003; Obuz et al., 2003; Panea et al., 2010). Normalmente, en la carne se utilizan temperaturas bajas, con tiempos largos en piezas grandes y cortos en piezas pequeñas, y un terminado final para la superficie (Kadam et al., 2015; Rocá et al., 2016). Así, por ejemplo, Rocá et al. (2016), teniendo en cuenta el gusto de los consumidores españoles, recomiendan $55^{\circ} \mathrm{C}$ para un solomillo al punto y $70^{\circ} \mathrm{C}$ para uno muy hecho. Pero en el rango $55^{\circ} \mathrm{C}-70^{\circ} \mathrm{C}$ ocurren en el músculo una gran cantidad de trasformaciones estructurales que afectan a la textura de la misma (Purslow, 1985; Palka y Daun, 1999; Christensen et al., 2000; Palka, 2003; Christensen et al., 2011b; Christensen et al., 2013; Purslow et al., 2016), ya que el calor solubiliza el colágeno y desnaturaliza las proteínas miofibrilares y sarcoplásmicas. Además, el grado en que la textura se ve afectada por el calentamiento varía en función del tiempo de maduración de la carne y de la raza (Palka, 2003; Kolczak et al., 2007; Panea et al., 2008b; Panea et al., 2010).

Por lo tanto, el objetivo del presente trabajo fue estudiar, en dos razas bovinas de aptitudes diferentes, los cambios que ocurren en la textura de la carne y la solubilidad del colágeno en función de la temperatura de cocinado y el tiempo de maduración.

\section{Material y métodos}

Se utilizaron 25 animales, 13 de raza Pirenaica y 12 de raza Frisona. La raza Pirenaica es una raza de aptitud cárnica, autóctona de la cordillera Pirenaica, integrada en el Catálogo oficial de razas de ganado (BOE, 2009) como raza autóctona de fomento (http://feagas.com/razas/bovino/pirenaica/). La raza Frisona es la raza lechera más extendida a nivel mundial y está reconocida en el Catálogo Oficial de Razas de España como una raza española integrada en España, ya que supone el $40 \%$ del censo total de dicha cabaña (http:// feagas.com/razas/bovino/frisona/). Por lo tanto, se eligieron estas dos razas como representantes de los dos grandes grupos de aptitud productiva del ganado vacuno: carne o leche. Los animales procedían de granjas comerciales y se compraron en los mataderos de Fribin, S.L. (Huesca), La Protectora (Pamplona) y Profesionales de la Carne, S.L. (Zaragoza).

Todos los animales presentaron un grado de engrasamiento 2 en la escala SEUROP, lo que implica que los animales de la raza Pirenaica se sacrificaron con 400 días de edad y un peso de canal fría de $173 \mathrm{Kg}$ y los animales de la raza Frisona se sacrificaron con 300 días de edad y $113 \mathrm{Kg}$ de canal fría.

A las 24 horas post-mortem se extrajeron los chuleteros de las medias canales izquierdas desde la $5^{a}$ vértebra torácica hasta el final de la zona lumbar, que fueron transportados en refrigeración al laboratorio del CITA. Se extrajo el $\mathrm{m}$. Longissimus thoracis et lumborum, se midió el pH en la parte craneal y se obtuvo un filete de $1 \mathrm{~cm}$ de espesor para la determinación de la cantidad total de colágeno en carne cruda. La muestra se envasó en una bolsa de vacío de polietileno de baja densidad y poliamida (LDPE/PA/LDPE, $75 \mathrm{~m}$ de espesor, permeabilidad al oxígeno de 52,2 $\mathrm{cm}^{3} / \mathrm{m}^{2}$ y día a $1 \mathrm{~atm}$ de presión, $75 \%$ de humedad relativa $(\mathrm{RH})$ y $23^{\circ} \mathrm{C}$ y una permeabilidad al vapor de agua de $2,4 \mathrm{~g} / \mathrm{m}^{2}$ y día a 
$\left.100 \% \mathrm{RH}, 23^{\circ} \mathrm{C}\right)$. Esta muestra se congeló a $-20^{\circ} \mathrm{C}$ con 1 día de maduración, manteniéndose así hasta el día del análisis.

El resto del lomo se fileteó siguiendo un diseño factorial con tres tiempos de maduración $(7,14$ y 21 días) y 4 temperaturas de cocinado para la textura (crudo, $55^{\circ} \mathrm{C}, 65^{\circ} \mathrm{C}$ y $70^{\circ} \mathrm{C}$ ), o 3 para el colágeno insoluble y las pérdidas por cocinado $\left(55^{\circ} \mathrm{C}, 65^{\circ} \mathrm{C}\right.$ y $\left.70^{\circ} \mathrm{C}\right)$. Las muestras se pesaron previamente al cocinado y todas las muestras sometidas a calor se cocinaron siempre dentro de la bolsa de vacío (con las características descritas arriba) y registrando la temperatura del filete mediante un termómetro Testo Datlogger 177-T4 con termopares tipo $\mathrm{T}$.

Para la determinación de la textura, se cortaron las muestras en forma de paralelepípedo con una sección de $1 \mathrm{~cm}^{2}$ y configura- ción longitudinal (Honikel, 1998). Para el ensayo se utilizó un Instron 5543 equipado con una célula de Warner-Bratzler (WB) y otra de compresión (Lepetit y Culioli, 1994). Se registraron el esfuerzo de cizalla en WB (esfuerzo WB) y la fuerza de compresión a tasas del $20 \%\left(C_{20}\right), 80 \%\left(C_{80}\right)$ y $100 \%\left(C_{100}\right)$ de la máxima compresión posible $\left(\mathrm{N} / \mathrm{cm}^{2}\right)$. La concentración de colágeno se determinó por el método de Bonnet y Kopp (1984). Se utilizó un coeficiente de conversión de 8 para la hidroxiprolina y los resultados se expresan como $\mathrm{mg}$ de colágeno/g de músculo.

Con los filetes de los ensayos de textura y colágeno se calcularon las pérdidas por cocinado, expresándose como (peso crudo-peso cocinado) x 100/peso crudo.

Un esquema del diseño puede verse en la Figura 1.

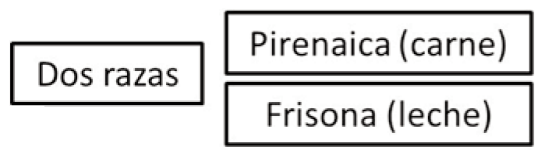

\begin{tabular}{|c|c|c|c|c|c|c|c|c|c|}
\hline & & \multicolumn{8}{|c|}{ Maduración } \\
\hline & & 1 día & & días & & días & & & días \\
\hline \multirow{8}{*}{ 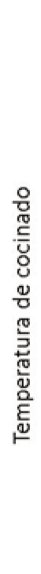 } & \multirow{2}{*}{ Crudo } & $\mathrm{pH}_{24}$ & WB & aresión & WB cor & esión & WB & con & resión \\
\hline & & Colágeno total & & & & & & & \\
\hline & \multirow[b]{2}{*}{$55^{\circ} \mathrm{C}$} & & & compresión & WB compresión & compresión & WB compresión & \multicolumn{2}{|c|}{ compresión } \\
\hline & & & \multicolumn{2}{|c|}{\begin{tabular}{l|l} 
Colageno & \%érdidas \\
\end{tabular}} & Colágeno & \%Pérdidas & & no & \%Pérdidas \\
\hline & \multirow[b]{2}{*}{$65^{\circ} \mathrm{C}$} & & WB & compresión & WB & compresión & WB & \multicolumn{2}{|c|}{ compresión } \\
\hline & & & Colágeno & \%Pérdidas & Colágeno & \%Pérdidas & \multicolumn{2}{|c|}{ Colágeno } & \%Pérdidas \\
\hline & \multirow[b]{2}{*}{$709 \mathrm{C}$} & & WB & compresión & WB & compresión & WB & \multicolumn{2}{|c|}{ compresión } \\
\hline & & & Colágeno & \%Pérdidas & Colágeno & \%Pérdidas & & & \%Pérdidas \\
\hline
\end{tabular}

Figura 1. Esquema del diseño experimental.

Figure 1. Experimental design. 


\section{Análisis estadísticos}

Los análisis se han realizado intra-raza, ya que la comparación racial no es el objetivo del presente trabajo. El efecto de la maduración y temperatura de cocinado sobre las variables de textura, la cantidad de colágeno insoluble y las pérdidas por cocinado se estudió por medio de un GML. Para todas las variables se calcularon las medias y error estándar. Las diferencias entre medias se establecieron con un test de Duncan $(p<0,05)$. Se utilizó el programa SPSS 13.0.

\section{Resultados}

El $\mathrm{pH}_{24}$ fue de 5,5 que se encuentra en el rango normal para la especie y permite descartar efectos sobre el resto de las variables estudiadas (Panea, 2002; Monsón et al., 2004; Panea et al., 2008a; Panea et al., 2010).

En la Tabla 1 se presenta la significación de los efectos estudiados sobre las variables de textura, colágeno insoluble y pérdidas por cocinado. En general, la maduración tuvo un efecto mucho menor que la temperatura. Así, sólo afectó significativamente al $C_{20}$ en la

Tabla 1. Significación (valor de p) de los efectos estudiados (tiempo de maduración y temperatura de cocinado) sobre las variables de textura, la cantidad de colágeno insoluble y las pérdidas por cocinado en carne de dos razas bovinas.

Table 1. $p$ values for studied effects (ageing time and cooking temperature) on texture variables, insoluble collagen amount and cooking losses in meat from two bovine breeds.

\begin{tabular}{|c|c|c|c|c|c|c|}
\hline & \multicolumn{3}{|c|}{ Pirenaica } & \multicolumn{3}{|c|}{ Frisona } \\
\hline & M & $T$ & $\mathrm{MxT}$ & M & $T$ & MxT \\
\hline Esfuerzo WB (N/cm²) & 0,070 & 0,102 & 0,748 & 0,108 & 0,017 & 0,774 \\
\hline $\mathrm{C}_{20}\left(\mathrm{~N} / \mathrm{cm}^{2}\right)$ & 0,013 & 0,000 & 0,038 & 0,789 & 0,103 & 0,860 \\
\hline $\mathrm{C}_{80}\left(\mathrm{~N} / \mathrm{cm}^{2}\right)$ & 0,230 & 0,004 & 0,400 & 0,513 & 0,004 & 0,234 \\
\hline $\mathrm{C}_{100}\left(\mathrm{~N} / \mathrm{cm}^{2}\right)$ & 0,284 & 0,020 & 0,341 & 0,346 & 0,005 & 0,469 \\
\hline Colágeno insoluble (mg/g músculo) & 0,084 & 0,000 & 0,189 & 0,348 & 0,000 & 0,017 \\
\hline Pérdidas por cocinado (\%) & 0,421 & 0,000 & 0,631 & 0,907 & 0,000 & 0,057 \\
\hline
\end{tabular}

M: tiempo de maduración; T: temperatura de cocinado; Esfuerzo WB: esfuerzo máximo de cizalla en WB; $C_{20}$ : fuerza de compresión a tasas del $20 \%$ de la máxima compresión posible; $\mathrm{C}_{80}$ : fuerza de compresión a tasas del $80 \%$ de la máxima compresión posible; $C_{100}$ : fuerza de compresión a tasas del $100 \%$ de la máxima compresión posible.

raza Pirenaica $(p=0,013)$ y se han encontrado interacciones entre efectos para el $\mathrm{C}_{20}$ en la raza Pirenaica $(p=0,038)$ y para la cantidad de colágeno insoluble en la raza Frisona $(p=0,017)$.

Sin embargo, la temperatura afectó a todas las variables excepto al esfuerzo WB en la raza Pirenaica $(p=0,102)$ y al $C_{20}$ en la raza Frisona $(p=0,103)$.

En la Figura 2 se representa gráficamente la evolución de los parámetros estudiados con el aumento de la temperatura. Aunque en la raza Pirenaica no hay efecto de la temperatura sobre el esfuerzo WB y en la raza Frisona 


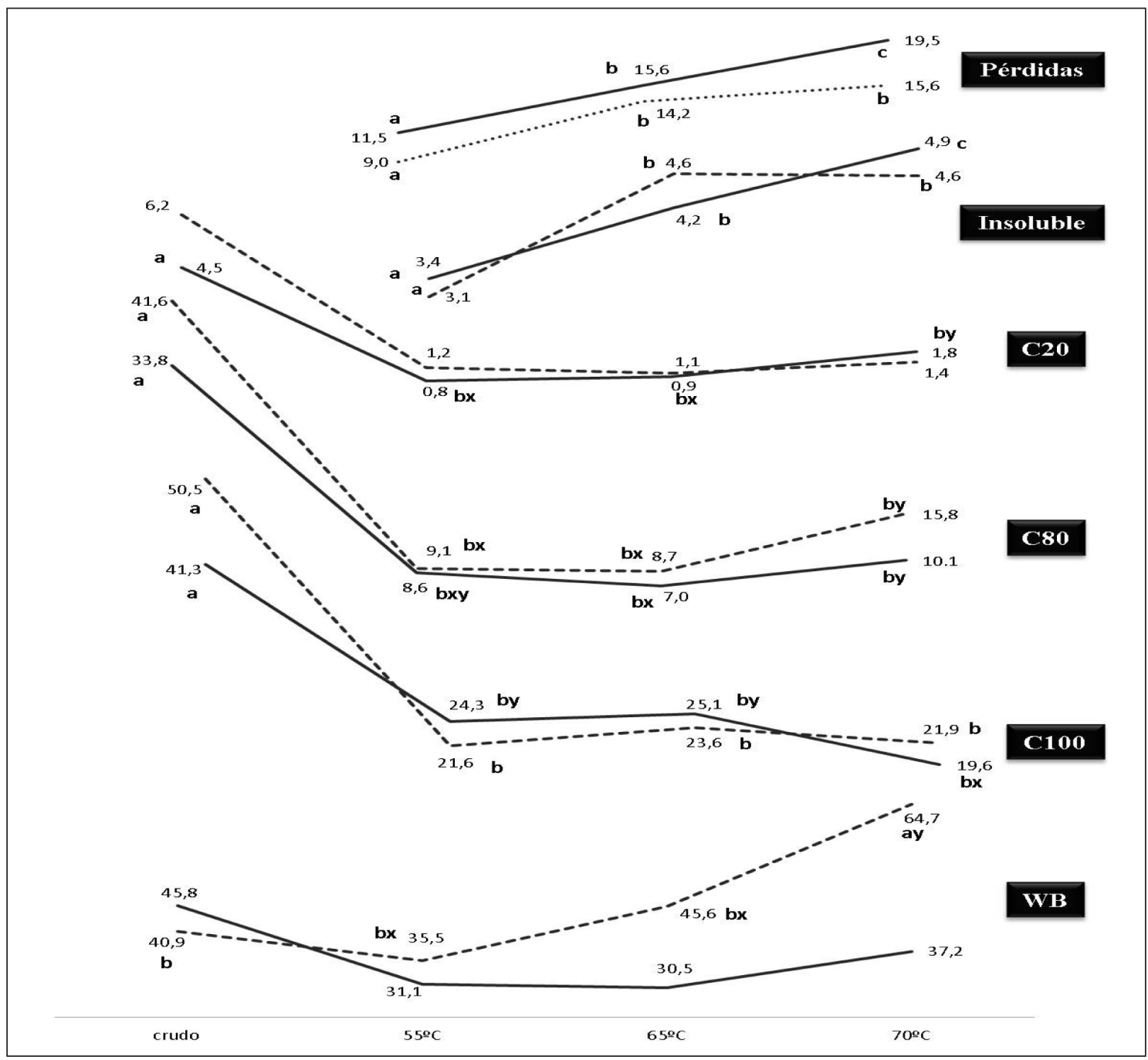

Figura 2. Influencia de la temperatura de cocinado sobre las variables de textura, la cantidad de colágeno insoluble y las pérdidas por cocinado en carne de dos razas bovinas. Los valores de cada variable aparecen junto a la línea correspondiente.

Línea continua: Pirenaica; Línea discontinua: Frisona; Insoluble: cantidad de colágeno insoluble (mg/g); pérdidas: porcentaje de pérdidas de peso por cocinado; WB: esfuerzo máximo de cizalla en WB; $\mathrm{C}_{20}$ : fuerza de compresión a tasas del $20 \%$ de la máxima compresión posible; $C_{80}$ : fuerza de compresión a tasas del $80 \%$ de la máxima compresión posible; $C_{100}$ : fuerza de compresión a tasas del $100 \%$ de la máxima compresión posible.

Variables de textura expresadas en $\mathrm{N} / \mathrm{cm}^{2} ; a$, b: diferencias entre temperaturas; $\mathrm{x}, \mathrm{y}$ : diferencias entre temperaturas sin contar la carne cruda.

Figure 2. Influence of cooking temperature on texture variables, insoluble collagen amount and cooking losses in meat from two bovine breeds. Values for each variable appear next to the correspondent line. 
no hay efecto de la temperatura sobre el $\mathrm{C}_{20}$, en la Figura 2 se han representado también estas variables para ayudar a la compresión de la evolución de la textura con la temperatura. Para poder representar todas las curvas en una sola figura, se han eliminado los ejes de abscisas y los valores de cada variable se presentan junto a cada curva. Las líneas continuas corresponden a la raza Pirenaica y las discontinuas, a la Frisona.

Los valores para las pérdidas por cocinado oscilan entre el $12 \%$ y el $20 \%$ en la Pirenaica (error estándar (e.e.) $=0,9$ ) y el $9 \%$ y $16 \%$ en la Frisona (e.e. $=0,7)$. En ambas razas, el porcentaje de pérdidas por cocinado va aumentando al hacerlo la temperatura pero mientras que en la raza Pirenaica lo hace de forma gradual, en la raza Frisona, hay un aumento entre $55^{\circ} \mathrm{C}$ y $65^{\circ} \mathrm{C}$, para estabilizarse después.

En ambas razas, la cantidad de colágeno insoluble varía muy poco en función de la temperatura en el rango estudiado (Figura 2). En la raza Pirenaica, la cantidad de colágeno insoluble aumenta paulatinamente al hacerlo la temperatura, pero en la raza Frisona se encontró una interacción maduracióntemperatura y así, en la Tabla 2 puede verse que en la raza Frisona hay un aumento paulatino con la temperatura en carne madurada 21 días, pero en la carne madurada 7 o 14 días, hay un pico máximo a los $65^{\circ} \mathrm{C}$. La cantidad de colágeno total en carne cruda fue de 5,2 mg/g en la raza Pirenaica y de 5,3 $\mathrm{mg} / \mathrm{g}$ en la Frisona (datos no tabulados), no existiendo diferencias entre razas $(p=0,446)$. Por lo tanto, el porcentaje de colágeno insoluble estaba siempre por encima del $60 \%$. En nuestro laboratorio, la metodología estándar para la determinación de colágeno insoluble se hace con carne madurada 1 día y cocinada a $90^{\circ} \mathrm{C}$. El dato es de $2,5 \mathrm{mg} / \mathrm{g}$ para la Pirenaica y de $2,4 \mathrm{mg} / \mathrm{g}$ para la Frisona, no existiendo diferencias entre razas $(p=0,496)$. Es decir, incluso a $90^{\circ} \mathrm{C}$, sigue habiendo un $45 \%$ de colágeno insoluble en la carne.

Hay un descenso del $C_{20}$ al pasar de carne cruda a carne cocinada a $55^{\circ} \mathrm{C}$, y se mantiene a partir de entonces. El comportamiento del $\mathrm{C}_{80}$ es similar en ambas razas y muy parecido al del $C_{20}$. En cuanto al $C_{100}$, existe en ambas razas un descenso acusado hasta $55^{\circ} \mathrm{C}$ y mantenimiento entre $55^{\circ} \mathrm{C}$ y $65^{\circ} \mathrm{C}$, pero a partir de $65^{\circ} \mathrm{C}$ desciende en la Pirenaica y se mantiene constante en la Frisona. Por último, el

Tabla 2. Medias para la cantidad de colágeno insoluble ( $\mathrm{mg} / \mathrm{g}$ músculo) y valor de $\mathrm{p}$ para los efectos estudiados (tiempo de maduración y temperatura de cocinado) en la carne de dos razas bovinas. Table 2. Means for insoluble collagen amount ( $\mathrm{mg} / \mathrm{g}$ muscle) and $p$ values for studied effects (ageing time and cooking temperature) in meat from two bovine breeds.

\begin{tabular}{|c|c|c|c|c|c|c|c|}
\hline & & \multicolumn{3}{|c|}{ Temperatura de cocinado } & \multicolumn{3}{|c|}{ Valor de $p$} \\
\hline & & M & T & MxT & M & T & MxT \\
\hline \multirow[t]{3}{*}{ Frisona } & 7 días & $2,93^{\mathrm{a}}$ & $4,63^{b}$ & $4,08^{b}$ & 0,384 & 0,000 & 0,017 \\
\hline & 14 días & 3,56 & 5,26 & 4,21 & & & \\
\hline & 21 días & $2,88^{a}$ & $3,97^{b}$ & $5,50^{c}$ & & & \\
\hline Pirenaica & & $3,35^{\mathrm{a}}$ & $4,21^{b}$ & $4,93^{c}$ & 0,084 & 0,000 & 0,189 \\
\hline
\end{tabular}

M: tiempo de maduración; T: temperatura de cocinado; ${ }^{a}, b, c$ : letras diferentes en la misma fila indican diferencias significativas entre temperaturas $(p<0,05)$. 
esfuerzo WB no se vio afectado por la temperatura de cocinado en la raza Pirenaica pero en la raza Frisona aumenta progresivamente a partir de los $55^{\circ} \mathrm{C}$. Las diferencias entre carne cruda y cocinada son tan grandes que enmascaran las diferencias debidas a la temperatura de cocinado. Si se elimina la carne cruda del análisis, el paso de $65^{\circ} \mathrm{C}$ a $70^{\circ} \mathrm{C}$ produce un ligero incremento en los valores de $C_{20}, C_{80}$ y esfuerzo WB y un ligero descenso en los valores de $C_{100}$.

\section{Discusión}

La carne tiene una estructura compleja y todos sus componentes contribuyen a la dureza de la misma. Sin embargo, cada uno de los componentes responde de distinta manera al calentamiento y a la maduración.

\section{Pérdidas por cocinado}

Los valores para las pérdidas por cocinado coinciden con los descritos por otros autores en las mismas razas (Sañudo et al., 1998; Martínez-Cerezo et al., 2001; Panea et al., 2008a; Barahona et al., 2016).

Aunque Palka (2003) describe que las pérdidas de agua son aproximadamente un 3\% mayores en carne madurada 12 días que en carne madurada 5 días, la mayoría de los autores señalan una falta de efecto de la maduración sobre las pérdidas por cocinado (Aaslyng et al., 2003; Straadt et al., 2007; Hughes et al., 2014).

El incremento de la pérdidas por cocinado con la temperatura ha sido ampliamente descrito en la bibliografía y puede encontrarse una buena revisión en Hughes et al. (2014). Según esta revisión, hacia los $65^{\circ} \mathrm{C}-66^{\circ} \mathrm{C}$ se detecta el cambio más importante en el flujo de agua dentro del filete, porque se aúnan la contracción del colágeno y la de las fibras mus- culares. Sin embargo, Purslow et al. (2016) concluyen que no hay evidencias de que el colágeno contribuya a aumentar el porcentaje de pérdidas cuando se contrae hacia los $65^{\circ} \mathrm{C}$ y por tanto, por encima de los $42^{\circ} \mathrm{C}$ las pérdidas se deben sobre todo a la contracción de las fibras musculares. La contracción longitudinal y transversal de la estructura muscular con el calentamiento provoca una reducción de volumen en el filete que puede llegar al $10 \%$ de su volumen y que origina la expulsión de agua libre, ya que aproximadamente el $80 \%$ del agua del músculo está ligada a las proteínas miofibrilares (Baldwin, 2012). En el presente trabajo el cambio fue gradual en la raza Pirenaica pero en la raza Frisona fue mucho más marcado entre $55^{\circ} \mathrm{C}$ y $65^{\circ} \mathrm{C}$. Así, si en la raza Frisona calculamos el incremento en las pérdidas como porcentaje sobre la diferencia máxima, el $79 \%$ del incremento se produce en el rango $55^{\circ} \mathrm{C}-65^{\circ} \mathrm{C}$.

\section{Colágeno insoluble}

Los valores de solubilidad del colágeno coinciden con los de otros autores en las mismas razas (Sañudo et al., 1998; Panea et al., 1999; Panea, 2002). En el presente trabajo no se encontró efecto de la maduración sobre la cantidad de colágeno insoluble, aunque algunos autores señalan que durante la maduración hay proteólisis del colágeno y esto incrementa la cantidad de colágeno que puede solubilizarse (Wu et al., 1985; Purslow, 2005). En el presente estudio se encontró una interacción entre temperatura y tiempo de maduración para la raza Frisona, lo que estaría de acuerdo con los resultados encontrados por Palka (2003), quien señala que cuando la maduración es corta (5 días) hay un aumento de la solubilidad entre $70^{\circ} \mathrm{C}$ y $80^{\circ} \mathrm{C}$, y un descenso entre $80^{\circ} \mathrm{C}$ y $90^{\circ} \mathrm{C}$ pero que cuando la maduración es larga (12 días) no hay cambios entre $80^{\circ} \mathrm{C}$ y $90^{\circ} \mathrm{C}$.

Los cambios en la estructura del endomisio y perimisio con el aumento de la temperatura 
están bien documentados en la bibliografía (Wu et al., 1985; Rowe, 1989; Palka y Daun, 1999). En la carne cruda no se observan grandes cambios en la estructura, pero a $60^{\circ} \mathrm{C}$ el endomisio pierde su aspecto fibroso y empieza a gelatinizarse. $\mathrm{A} 70^{\circ} \mathrm{C}$ se completa la contracción del endomisio y a $80^{\circ} \mathrm{C}$ el perimisio aparece coagulado. A $90^{\circ} \mathrm{C}$, la estructura se vuelve amorfa. Algunos estudios de calorimetría han demostrado que hay un pico endotérmico a $68^{\circ} \mathrm{C}$ y otro a $77^{\circ} \mathrm{C}$, es decir, que a esas temperaturas hay algún cambio conformacional en el músculo pero sigue sin estar claro cuánto colágeno sigue sin desnaturalizar por debajo de $68^{\circ} \mathrm{C}$ (Christensen et al., 2013). Por lo tanto, debería esperarse un incremento de la solubilidad con el aumento de la temperatura, lo que no ocurre en nuestro experimento. Este desacuerdo podría explicarse en parte por las condiciones de solubilización empleadas. En la mayoría de los trabajos la cantidad de colágeno soluble se determina en carne picada y con una solución tamponada de $\mathrm{pH}$ neutro, pero en el presente trabajo el filete se cocinó entero y sin líquido de gobierno, excepto la propia agua expelida por el filete. En estas condiciones y a partir de nuestros resultados, cabe plantear la hipótesis de que la contracción de la estructura miofibrilar y la consiguiente pérdida de agua libre, así como la degradación de las proteínas sarcoplásmicas, que se completa hacia los $60^{\circ} \mathrm{C}$, está afectando a la cantidad de colágeno que se puede extraer del músculo. En cierto modo es como si estos cambios conformacionales protegiesen al colágeno de la solubilización y por eso hay un aumento de la cantidad de colágeno insoluble entre $55^{\circ} \mathrm{C}$ y $75^{\circ} \mathrm{C}$. Sin embargo, los datos obtenidos en carne cruda a $90^{\circ} \mathrm{C}$, que se realizaron en carne picada siguiendo el procedimiento habitual del laboratorio (Bonnet y Kopp, 1984) dejan claro que a temperaturas altas el músculo está completamente degradado y el agua expulsada actúa como solución. Esta idea vendría reforzada por los es- tudios de Powell et al. (2000) que aportan dos conclusiones importantes: a) la fracción de colágeno soluble en una solución Ringer no aumenta con el incremento de temperatura en el rango $50^{\circ} \mathrm{C}-70^{\circ} \mathrm{C}$ y b) a $50^{\circ} \mathrm{C}$, el $70 \%$ del colágeno no puede extraerse ni mediante una solución Ringer ni mediante métodos enzimáticos y a $70^{\circ} \mathrm{C}$ esta fracción representa todavía el $23 \%$ del colágeno del músculo.

\section{Textura}

Los valores encontrados para las variables de textura coinciden con los descritos por otros autores en las mimas razas (Campo et al., 1998; Sañudo et al., 1998; Lepetit et al., 2000; Martínez-Cerezo et al., 2001; Panea, 2002; Monsón et al., 2004).

Como era esperable, el efecto de la maduración fue menos importante que el de la temperatura de cocinado ya que el efecto de la maduración sobre el $\mathrm{C}_{20}$ es más importante sobre todo en la primera semana de maduración, representando alrededor del $65 \%$ en la primera semana y del $11 \%$ en la segunda ( $\mathrm{Pa}$ nea et al., 2008b). Durante la maduración hay un debilitamiento progresivo del perimisio debido a la degradación de los proteoglicanos, lo cual puede hacer que la dureza se reduzca alrededor del $30 \%$ en 14 días en carne cruda (Wu et al., 1985) pero este efecto de reducción desaparece cuando se cocina la carne a temperaturas de $60^{\circ} \mathrm{C}$ o más, ya que entonces la fuerza que ejerce el perimisio es independiente de la maduración (Purslow, 2005).

Palka (2003) demostró en un trabajo con el músculo semitendinoso que existe una interacción maduración-temperatura para el esfuerzo WB. Así, observó un aumento de la dureza con la temperatura, pero mientras que este aumento se producía en maduraciones cortas a partir de los $60^{\circ} \mathrm{C}$, había que llegar a los $80^{\circ} \mathrm{C}$ en maduraciones largas. Esto explicaría que en nuestro experimento el incre- 
mento en $\mathrm{C}_{100}$ se produzca antes en la carne madurada 7 o 14 días que en la carne madurada 21 días.

La temperatura de cocinado tuvo un efecto claro sobre la textura de la carne, con un descenso marcado del $C_{20}$ y $C_{80}$ y un aumento del esfuerzo WB. Se ha señalado que cuando la ratio de compresión es menor del $40 \%$, los valores del esfuerzo muestran la influencia del componente miofibrilar sobre la textura y cuando la ratio es mayor del $80 \%$, el esfuerzo se debe a la contribución del tejido conjuntivo, pero estas conclusiones se refieren a la carne cruda, que es como suelen realizarse los ensayos con células de compresión (Lepetit, 1991; Lepetit y Culioli, 1994; Lepetit, 2008). Sin embargo, el cocinado modifica toda la estructura muscular.

Las proteínas miofibrilares y el colágeno se contraen cuando la carne se cocina, pero las proteínas sarcoplásmicas se expanden. La agregación y gelatinización de las proteínas sarcoplásmicas comienza hacia los $40^{\circ} \mathrm{C}$ y termina alrededor de los $60^{\circ} \mathrm{C}$, reduciendo la dureza de la carne (Baldwin, 2012). La contribución del colágeno a la dureza es mayor a temperaturas inferiores a la de solubilización, proceso que comienza hacia los $65^{\circ} \mathrm{C}$. Justo antes de la solubilización se produce un fenómeno de contracción térmica del colágeno y una compactación de las fibras musculares. Esta contracción provoca que el ángulo que forman el perimisio y la fibra muscular, que es de unos $55^{\circ} \mathrm{C}$ en el músculo en reposo, pase a ser de unos $75^{\circ} \mathrm{C}$ y eso aumenta su dureza (Grajales et al., 1999) Sin embargo, si calentamos las muestras por encima de la temperatura de contracción, el colágeno se solubiliza y se convierte en gelatina, lo que hace disminuir su resistencia mecánica (Grajales et al., 1999). En consecuencia, a partir de los $60^{\circ} \mathrm{C}$ la resistencia se debe principalmente a la miofibrilla (Purslow, 2005). Cuando se calienta la carne, se solubi- lizan las proteínas miofibrilares y esta solubilización aumenta con la temperatura de cocinado, de modo que a $70^{\circ} \mathrm{C}$ es un $8 \%$ mayor que a $55^{\circ} \mathrm{C}$ y a $75^{\circ} \mathrm{C}$, un $92 \%$ mayor que a $55^{\circ} \mathrm{C}$ (Kopp y Valin, 1979). Aún más, las proteínas de los filamentos gruesos y delgados responden de diferente manera al calentamiento en el rango $40^{\circ} \mathrm{C}-80^{\circ} \mathrm{C}$. La alfaactinina se desnaturaliza a $50^{\circ} \mathrm{C}$; la miosina a $55^{\circ} \mathrm{C}$; las proteínas del sarcoplasma hacia $65^{\circ} \mathrm{C}$; la titina a los $73^{\circ} \mathrm{C}$; la actina entre $70^{\circ} \mathrm{C}$ $80^{\circ} \mathrm{C}$, la tropomiosina y la troponina por encima de $80^{\circ} \mathrm{C}$ y la nebulina permanece intacta por encima de esta temperatura (Palka y Daun, 1999). Además, durante el calentamiento hay una contracción longitudinal que implica el progresivo acortamiento de los sarcómeros y un cambio en el ángulo formado entre el colágeno y la miofibrilla y todos estos cambios se resumen en un aumento de la dureza (Grajales et al., 1999; Palka y Daun, 1999; Lepetit et al., 2000; Panea et al., 2010).

En el presente experimento, hemos encontrado un descenso para las variables de textura hasta los $60^{\circ} \mathrm{C}$, lo que coincide con los resultados de otros autores (Combes et al., 2003; Panea et al., 2010; Christensen et al., 2011a; Baldwin, 2012). Nuestros resultados parecen indicar que en el rango $55^{\circ} \mathrm{C}-65^{\circ} \mathrm{C}$, el colágeno se está solubilizando y no ejerce un efecto notable sobre la textura de la carne, ya que no se detectan cambios en ninguno de los parámetros estudiados. La falta de correlación entre las características del colágeno y las de textura ya había sido descrita en la bibliografía (Liu et al., 1996; Christensen et al., 2011b). Por otro lado, en el rango $65^{\circ} \mathrm{C}-70^{\circ} \mathrm{C}$ se duplican los valores de $\mathrm{C}_{20}$ en la raza Pirenaica, los valores de $\mathrm{C}_{80}$ se incrementan en un $80 \%$ en la raza Frisona y en un $44 \%$ en la raza Pirenaica y el esfuerzo WB se incrementa en un $41 \%$ en la raza Frisona, lo que coincide con trabajos previos de nuestro laboratorio (Panea et al., 1999; Panea, 2002; Panea et al., 2008b; Panea et al., 2010). 


\section{Conclusiones}

La maduración tuvo un efecto menor que la temperatura de cocinado sobre la textura de la carne. El porcentaje de pérdidas por cocinado aumenta al hacerlo la temperatura. La solubilidad del colágeno se ve poco afectada por la temperatura en el rango $50^{\circ} \mathrm{C}-70^{\circ} \mathrm{C}$. En general, la dureza del músculo desciende hasta los $55^{\circ} \mathrm{C}$ para incrementarse ligeramente a partir de entonces. A la vista de los resultados, no recomendaríamos cocinar la carne por encima de $65^{\circ} \mathrm{C}$.

\section{Agradecimientos}

Este trabajo ha sido financiado por el proyecto INIA RTA2013-0046-C03. Los autores quieren agradecer el trabajo realizado por Carolina Albertí y Rocío Elías en el laboratorio.

\section{Referencias bibliográficas}

Aaslyng MD, Bejerholm C, Ertbjerg P, Bertram HC, Andersen HJ (2003). Cooking loss and juiciness of pork in relation to raw meat quality and cooking procedure. Food Quality and Preference 14: 277-288.

Baldwin DE (2012). Sous vide cooking: A review. International Journal of Gastronomy and Food Science 1: 15-30.

Barahona M, Olleta JL, Sañudo C, Albertí P, Panea B, Pérez-Juan M, Realini CE, Campo MM (2016). Effects of whole linseed and rumen-protected conjugated linoleic acid enriched diets on beef quality. Animal 10: 709-717.

BOE (2009). Real Decreto 2129/2008, de 26 de diciembre, por el que se establece el Programa nacional de conservación, mejora y fomento de las razas ganaderas. Boletín Oficial del Estado, núm. 23, de 27 de enero de 2009, pp. 9211-9242.

Bonnet M, Kopp J (1984). Dossage du collagene dans les tissues conjonctifs, la viande et les produits carnes. Cahier Techniques INRA 5: 19-30.
Campo MM, Sañudo C, Panea B, Albertí P, Santolaria $P$ (1998). Breed and ageing time effects on textural sensory characteristics of beef strip loin steaks. Proceedings of 44th International Congress of Meat Science and Technology: Meat Consumption and Culture. August 30th September 4th, Barcelona, Spain.

Combes S, Lepetit J, Darche B, Lebas F (2003). Effect of cooking temperature and cooking time on Warner-Bratzler tenderness measurement and collagen content in rabbit meat. Meat Science 66: 91-96.

Christensen L, Ertbjerg P, Aaslyng MD, Christensen $M$ (2011a). Effect of prolonged heat treatment from 48 degrees $C$ to 63 degrees $C$ on toughness, cooking loss and color of pork. Meat Science 88: 280-285.

Christensen L, Ertbjerg P, Løje H, Risbo J, van den Berg FW, Christensen M (2013). Relationship between meat toughness and properties of connective tissue from cows and young bulls heat treated at low temperatures for prolonged times. Meat Science 93: 787-795.

Christensen M, Purslow PP, Larsen LM (2000). The effect of cooking temperature on mechanical properties of whole meat, single muscle fibres and perimysial connective tissue. Meat Science 55: 301-307.

Christensen M, Ertbjerg P, Failla S, Sanudo C, Richardson RI, Nute GR, Olleta JL, Panea B, Alberti $\mathrm{P}$, Juarez $\mathrm{M}$, Hocquette JF, Williams JL (2011b). Relationship between collagen characteristics, lipid content and raw and cooked texture of meat from young bulls of fifteen European breeds. Meat Science 87: 61-65.

Grajales A, Lepetit J, Favier R (1999). Geometrical modifications of perimysial connective tissue in meat during cooking. Proceedings of $45 \mathrm{th}$ International Congress of Meat Science and Technology: Meat joins countries around the world August 1-6. Yokohama, Japan. pp.522-523.

Honikel KO (1998). Reference methods for the assessment of physical characteristics of meat. Meat Science 49: 447-457.

Hughes JM, Oiseth SK, Purslow PP, Warner RD (2014). A structural approach to undestanding the inter- 
actions between colour, water-holding capacity and tenderness. Meat Science 98: 520-532.

Kadam SU, Tiwari BK, O'Donnell CP (2015). 6 - Improved thermal processing for food texture modification A2. En: Modifying Food Texture (Ed. Chen J y Rosenthal A) pp. 115-131. Woodhead Publishing.

Kolczak T, Krzysztoforski K, Palka K (2007). The effect of post-mortem ageing and heating on water retention in bovine muscles. Meat Science 75: 655-660.

Kopp J, Valin C (1979). Evolution post mortem des propietés de contraction thermique du muscle de bovin. Annales de Technologie Agricole 28: 107-119.

Lepetit J (1991). Theoretical strain ranges in raw meat. Meat Science 29: 271-283.

Lepetit J, Culioli J (1994). Mechanical properties of meat. Meat Science 36: 203-237.

Lepetit J, Grajales A, Favier R (2000). Modelling the effect of sarcomere length on collagen thermal shortening in cooked meat: consequence on meat toughness. Meat Science 54: 239-250.

Lepetit J (2008). Collagen contribution to meat toughness: Theoretical aspects. Meat Science 80: 960-967.

Martínez-Cerezo S, Sañudo C, Olleta JL, Panea B, Serrano X, Castrillo C (2001). Efecto del peso al sacrificio, el sexo y el extrusionado de la dieta sobre la calidad sensorial de la carne en terneros de raza Frisona. ITEA-Informacion Tecnica Economica Agraria Vol. Extra 22: 571-573.

Monsón F, Sañudo C, Sierra I (2004). Influence of cattle breed and ageing time on textural meat quality. Meat Science 68: 595-602.

Obuz E, Dikeman ME,. Loughin TM (2003). Effects of cooking method, reheating, holding time, and holding temperature on beef longissimus lumborum and biceps femoris tenderness. Meat Science 65: 841-51.

Palka K, Daun H (1999). Changes in texture, cooking losses, and myofibrillar structure of bovine $M$. semitendinosus during heating. Meat Science 51: 237-243.
Palka K (2003). The influence of post-mortem ageing and roasting on the microstructure, texture and collagen solubility of bovine semitendinosus muscle. Meat Science 64: 191-198.

Panea B, Olleta JL, Beltrán JA, Sañudo C, Campo MM. (1999). Influence of breed in thermal stability of intramuscular bovine collagen. Proceedings of 45th International Congress of Meat Science and Technology: Meat joins countries around the world August 1-6. Yokohama, Japan.

Panea B (2002). Influencia de la raza-sistema productivo sobre el tejido conjuntivo y la textura de la carne bovina. Tesis Doctoral. Universidad de Zaragoza. 226 pp.

Panea B, Albertí P, Olleta JL, Campo MM, Ripoll G, Altarriba J, Sañudo C (2008a). Intrabreed variability and relationships for 41 carcass and meat traits in Pirenaica cattle. Spanish Journal of Agricultural Research 6: 546-558.

Panea B, Sañudo C, Olleta JL, Civit D (2008b). Effect of ageing method, ageing period, cooking method and sample thickness on beef textural characteristics. Spanish Journal of Agricultural Research 6: 25-32.

Panea B, Catalán A, Olleta JL (2010). Efecto de la raza y temperatura interna de cocinado sobre algunas carcaterísticas de la textura de la carne bovina. ITEA-Informacion Tecnica Economica Agraria 106: 77-88.

Powell TH, Hunt MC, Dikeman ME (2000). Enzymatic assay to determine collagen thermal denaturation and solubilization. Meat Science 54: 307-311.

Purslow PP (1985). The physical basis of meat texture: Observations on the fracture behavior of cooked bovine $M$. Semitendinosus. Meat Science 12: 39-60.

Purslow PP (2005). Intramuscular connective tissue and its role in meat quality. Meat Science 70 : 435-447.

Purslow PP, Oiseth S, Hughes J, Warner RD (2016). The structural basis of cooking loss in beef: Variations with temperature and ageing. Food Research International 89: 739-748. 
Rocá J, Universa, Girona Ud (2016). Introducción a la cocina al vacío de El Celler de Can Roca.

Rowe RWD (1989). Microscopy of bovine muscle: II. The effects of heat denaturation on post rigor sarcolema and endomisium. Meat Science 26: 281-294.

Sañudo C, Albertí P, Campo MM, Olleta JL, Panea B (1998). Calidad instrumental de la carne de bovino de siete razas españolas. Archivos de Zootecnia 48: 397-402.

Straadt IK, Rasmussen M, Andersen HJ, Bertram HC (2007). Aging-induced changes in microstructure and water distribution in fresh and cooked pork in relation to water-holding capacity and cooking loss - A combined confocal laser scanning microscopy (CLSM) and low-field nuclear magnetic resonance relaxation study. Meat Science 75: 687-95.

Wu FY, Dutson TR, Smith SB (1985). A scanning electron microscopic study of heat-induced alterations in bovine connective tissue. Journal of Food Science 50: 1041-1044.

(Aceptado para publicación el 20 de noviembre de 2018) 